\title{
In Vivo Preclinical Molecular Imaging of Repeated Exposure to an $N$-methyl-D-aspartate Antagonist and a Glutaminase Inhibitor as Potential Glutamatergic Modulators ${ }^{[\$}$
}

\author{
Stijn Servaes, Firat Kara, Dorien Glorie, Sigrid Stroobants, Annemie Van Der Linden, \\ and Steven Staelens
}

Molecular Imaging Center Antwerp (Sti.S., D.G., Si.S., Ste.S.) and Bio-Imaging Laboratory (F.K., A.V.D.L.), University of Antwerp, Wilrijk, Antwerp, Belgium; and Department of Nuclear Medicine, University Hospital Antwerp, Edegem, Antwerp, Belgium (Si.S.)

Received August 3, 2018; accepted November 19, 2018

\begin{abstract}
Glutamate is the principal excitatory neurotransmitter in the brain and is at the base of a wide variety of neuropathologies, including epilepsy, autism, Fragile $\mathrm{X}$, and obsessive compulsive disorder. Glutamate has also become the target for novel drugs in treatment and in fundamental research settings. However, much remains unknown on the working mechanisms of these drugs and the effects of chronic administration on the glutamatergic system. This study investigated the chronic effects of two glutamate-modulating drugs with imaging techniques to further clarify their working mechanisms for future research opportunities. Animals were exposed to saline (1 $\mathrm{ml} / \mathrm{kg}), \quad(5 S, 10 R)-(+)-5-M e t h y l-10,11-d i h y d r o-5 H$-dibenzo $[a, d]$ cyclohepten-5,10-imine (MK-801) $(0.3 \mathrm{mg} / \mathrm{kg})$, or ebselen $(10 \mathrm{mg} / \mathrm{kg})$ for 7 consecutive days. At the sixth injection, animals underwent a positron emission tomography (PET)/ computed tomography (CT) with (3-(6-methyl-pyridin-2ylethynyl)-cyclohex-2-enone-O-11C-methyl-oxime) (ABP-688) to visualize the metabotropic $G$ protein-coupled glutamate
\end{abstract}

receptor 5 (mGluR5). After the seventh injection, animals underwent a magnetic resonance spectroscopy (MRS) scan to visualize glutamate and glutamine content. Afterward, results were verified by mGluR5 immunohistochemistry (IHC). PET/CT analysis revealed that animals receiving chronic MK-801 or ebselen had a significant $(P<0.05)$ higher binding potential $(2.90 \pm 0.47$ and $2.87 \pm 0.46$, respectively) when compared with saline $(1.97 \pm 0.39)$ in the caudate putamen. This was confirmed by mGluR5 IHC, with $60.83 \% \pm 6.30 \%$ of the area being highlighted for ebselen and $57.14 \% \pm 9.23 \%$ for MK- 801 versus $50.21 \% \pm 5.71 \%$ for the saline group. MRS displayed significant changes on the glutamine level when comparing chronic ebselen $(2.20 \pm 0.40 \mu \mathrm{mol} / \mathrm{g})$ to control $(2.72 \pm 0.34 \mu \mathrm{mol} / \mathrm{g})$. Therefore, although no direct effects on glutamate were visualized, the changes in glutamine suggest changes in the total glutamate-glutamine pool. This highlights the potential of both drugs to modulate glutamatergic pathologies.

\section{Introduction}

Glutamate is the principal excitatory neurotransmitter in the brain. It is transmitted via three families of ionotropic receptors, which have intrinsic cation permeable channels

This work was supported by a DocPro from the University of Antwerp [41/FA020000/FFB140317] and the Fonds Wetenschappelijk Onderzoek (FWO) from the University of Antwerp [42/FA020000/685]. This work was also funded by the University of Antwerp through a grant for Sti.S. and D.G., an assistant professor position for J. Verhaeghe, and a full professor position for Ste.S. and Si.S. Si.S. was also supported by Antwerp University Hospital, Belgium, through a departmental position. F.K. is a holder of an FWOVlaanderen (Belgium) postdoctoral fellowship (postdoctoral FWO number $12 \mathrm{~S} 4815 \mathrm{~N})$. Hardware and experimental costs were supported by a DOCPRO (41/FA020000/FFB140317) and an FWO KAN (42/FA020000/685) of Antwerp University. No funding sources had a role in the study design or in the collection, analysis, and interpretation of the data.

https://doi.org/10.1124/jpet.118.252635.

S This article has supplemental material available at jpet.aspetjournals.org.
[ $N$-methyl-D-aspartate (NMDA), $\alpha$-amino-3-hydroxy-5-methyl4-isoxazolepropionic acid, and kainite]. There are also three groups of metabotropic $\mathrm{G}$ protein-coupled glutamate receptors (mGluRs) that act on membrane ion channels and second messengers such as cAMP and diacylglycerol to modify neuronal and glial excitability. Additionally, there are three neuronal glutamate transporters in the brain and two glial glutamate transporters: the glial glutamate and aspartate transporter and the glial glutamate transporter (Meldrum, 2000).

It has been shown that overstimulation of the glutamatergic neurotransmission is neurotoxic (Porciúncula et al., 2001), and glutamate abnormalities have been well characterized in a number of neuropathologies, such as Fragile X, autism, schizophrenia, and epilepsy. Also, in other disorders, such as depression, anxiety, and more recently obsessive compulsive disorder (OCD), the emerging role of glutamate signaling has been garnering interest (Coyle, 2006; Pittenger et al., 2011;

ABBREVIATIONS: ABP-688, (3-(6-methyl-pyridin-2-ylethynyl)-cyclohex-2-enone-O-11C-methyl-oxime); BPnd, binding potential; CP, caudate putamen; Cr, creatine; CRLB, Cramér-Rao lower bounds; CT, computed tomography; Glu, glutamate; GPC, glycerophosphorylcholine; IHC, immunohistochemistry; mGluR, metabotropic G protein-coupled glutamate receptor; MK-801, (5S,10R)-(+)-5-Methyl-10,11-dihydro-5H-dibenzo [a,d]cyclohepten-5,10-imine; MR, magnetic resonance; MRS, magnetic resonance spectroscopy; NMDA, $N$-methyl-D-aspartate; OCD, obsessive compulsive disorder; PCr, phosphocreatine; PET, positron emission tomography; QP, quinpirole; VOI, volume of interest. 
Miladinovic et al., 2015). In addition, our group has previously shown that there is a role for glutamate in the quinpirole (QP) rodent model of OCD. In this model, animals are exposed to injections of QP and an open field test on a biweekly basis for a total duration of 5 weeks. These animals showed excessive compulsive behavior and were characterized by an increased distribution of mGluR5, as visualized by positron emission tomography (PET) (Servaes et al., 2017). Another group showed that in Sapap3 knockout mice, another preclinical model for OCD (Welch et al., 2007), mGluR5 signaling was elevated and associated with constitutively active postsynaptic receptors (Ade et al., 2016). As it is widely accepted that OCD results from a dysfunctional cortico-striato-thalamic circuitry (Fig. 1) (Denys et al., 2010), it is possible that, because the brain is exposed to higher levels of glutamate in OCD patients (Chakrabarty et al., 2005), the normal feedback loop is disrupted. Whether this is a consequence or a cause of the symptoms remains to be elucidated. Also, in other glutamate-related pathologies, such as Fragile $\mathrm{X}$ syndrome, autism, and epilepsy, there appears to be a specific role for the mGluR5 receptor (Pop et al., 2014; Chana et al., 2015; Kelly et al., 2018).

Taken together, glutamate has become a target for novel drugs. Therefore, drugs that can interact with the glutamatergic transmission, directly affecting the glutamate levels or the associated receptors in a positive or negative direction, could be of great assistance to further understand these pathologies. By modifying the glutamatergic system in either direction in a preclinical setting, more insight could be acquired in the manifestation of the pathologies mentioned before, either improving or aggravating the symptoms.

A variety of drugs interact with the glutamatergic system. Ebselen [2-phenyl-1,2-benzisoselenazol-3(2H)-one] is a selenoorganic compound of low toxicity that is characterized by a unique pharmacological profile (Schewe, 1995). Ebselen interacts directly with the glutamatergic system in rats (Nogueira et al., 2002). Although the exact mechanism of action has not been described yet (Azad and Tomar, 2014), preliminary results indicate that ebselen protects neurons from ischemic damage (Seo et al., 2009). Furthermore, in vitro studies have shown that ebselen inhibited $\left[{ }^{3} \mathrm{H}\right]$ glutamate release and uptake (Porciúncula et al., 2001). Importantly, ebselen is also an effective glutaminase inhibitor (Thomas et al., 2013). With glutaminase catalyzing the hydrolysis of glutamine to glutamate, this is a further indication of the direct effects of ebselen on the glutamatergic signal.

Another major group of glutamate-affecting drugs target the NMDA ionotropic glutamate receptor. NMDA receptors play a role in a variety of glutamate-associated pathologies, such as OCD (Arnold et al., 2004; Albelda et al., 2010), epilepsy (Ghasemi and Schachter, 2011), and autism (Lee et al., 2015). By directly modulating the NMDA receptors with NMDA receptor antagonists such as ketamine and (+)-MK-801 (dizocilpine maleate), [(5S,10R)-(+)-5-Methyl-10,11-dihydro5 H-dibenzo[a,d]cyclohepten-5,10-imine], the interneuron GABAergic receptors can be inhibited, which typically results in strong psychostimulatory and psychotomimetic effects. Furthermore, this modification could prove to be useful for investigating the NMDA abnormalities on the level of the receptor in the diseases mentioned earlier.

Ebselen's glutamate-reducing properties, together with its glutaminase inhibition and protection of ischemic damage, highlight its application potential for OCD. Additionally,

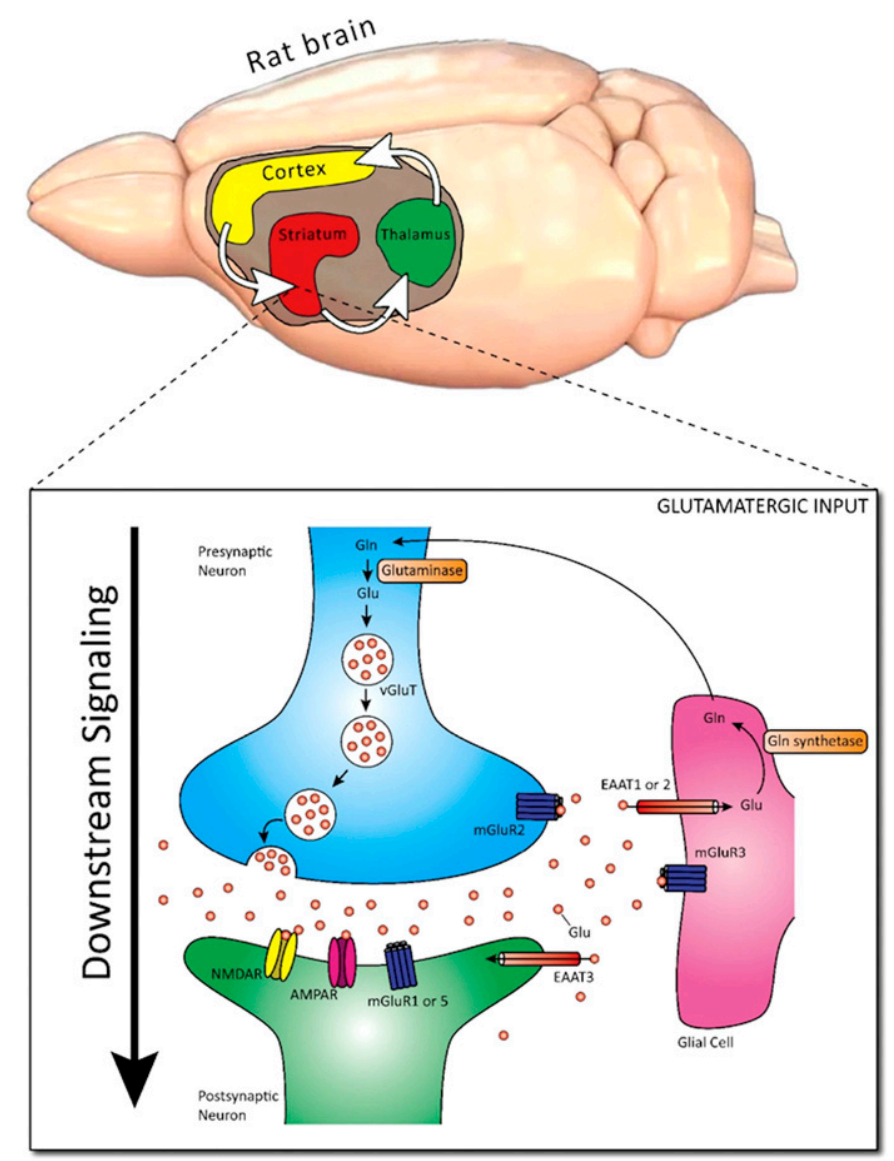

Fig. 1. The frontal cortex, responsible for functions such as error detection, working memory, and goal-directed behavior, sends signals through the striatum through glutamatergic afferents. From the striatum, this signal either inhibits or reduces the inhibition to the thalamus, depending on the levels of Dopamine present. From the thalamus, a signal is sent back to the frontal cortex, completing a feedback loop. It has been hypothesized that OCD results from a dysfunction in this circuit, in particular in the downstream signaling pathway. As the brain is exposed to higher levels of glutamate, this will likely cause overstimulation and disruption of the circuits and receptors in place. AMPAR, AMPA ( $\alpha$-amino3-hydroxy-5-methyl-4-isoxazolepropionic acid) receptor; NMDAR, NMDA receptor; vGluT, vesicular glutamate transporter.

although MK-801 will unlikely end up being a treatment option, the possibility for it to modulate glutamate levels could be of particular importance to further elucidate the underlying glutamatergic role in QP-induced OCD rats. Previously, MK-801 has been used in animal models for OCD to document the effect of activation of NMDA receptors on compulsions. Shannon and Love (2004) reported that MK-801 increased the rate of lever pressing in rats. In another animal model, MK-801 aggravated a transgene-dependent abnormal behavior likely due to the fact that it indirectly stimulates corticallimbic glutamate output (McGrath et al., 2000). Although a number of studies have looked at the acute effects of these drugs and their effect on glutamate release and the mGluR5 receptor, the result of chronic administration on neurotransmitter and receptor level remains elusive. Therefore, it is of critical importance to have an understanding of the effect of these drugs in a healthy animal to assess and influence their ability to modify the glutamatergic system. Ketamine, on the other hand, has previously been used in a treatment setting in OCD, with promising results (Bloch et al., 2012; Rodriguez et al., 2013). 
However, previous research has shown that the underlying working mechanisms of ketamine may be unique to ketamine and thus not shared by all NMDA receptor antagonists (Hillhouse and Porter, 2014). Indeed, ketamine's mechanism of action remains mysterious, as next to its NMDA blockade, ketamine disturbs a wide range of other intracellular neuronal processes (Sleigh et al., 2014).

In this study, we evaluated the potential of both MK- 801 and ebselen to modulate glutamate receptors and neurotransmitter levels in healthy naïve rats by means of magnetic resonance spectroscopy (MRS), PET, and immunohistochemistry (IHC). This allowed us to investigate the effect on 1) the neurotransmitter level by visualization of glutamate and glutamine with MRS and 2) on the neuroreceptor level by visualizing the receptor availability of mGluR5 and distribution by PET, as well as to verify this with mGluR5 IHC. For future preclinical research, such adds to elucidating the role of glutamate in neuropathologies such as OCD, in particular in the QP model discussed earlier, to establish whether modifying the glutamate levels has an effect on the symptoms.

\section{Materials and Methods}

The present study was carried out in accordance with the European Communities Council Directive of November 24th, 1986 (86/609/EEC) for the care of laboratory animals and after approval of the local ethical committee (University of Antwerp under number 2014-18). All efforts were made to minimize suffering and to reduce the number of animals. Naïve male Sprague-Dawley rats $(n=32,285-550 \mathrm{~g}$ during the experiment; Harlan, Netherlands) were housed in a temperatureand humidity-controlled vivarium in individually ventilated cages with a 12-hour light-dark cycle. All experiments were performed during daytime. Food and water were available ad libitum. All applicable institutional and national guidelines for the care and use of animals were followed.

Experimental Setup. Rats were divided into three groups. Animals in the first group $(n=12)$ received an intraperitoneal injection of MK-801 ( $0.3 \mathrm{mg} / \mathrm{kg}$; dissolved in saline) every day at the same time (5-6 PM) for 7 consecutive days. This dosage was chosen based on previous work using MK-801 to induce glutamate toxicity (Kosten et al., 2016) and an acute study investigating a variety of dosages (Wyckhuys et al., 2013). Animals in the second group $(n=12)$ were exposed to ebselen $(10 \mathrm{mg} / \mathrm{kg}$; dissolved in $0.5 \%$ carboxymethylcellulose) by oral gavage at the same time for 7 consecutive days also. The dosage of ebselen was based on previous studies (Dalla Corte et al., 2012; Yatmaz et al., 2013; Ozyigit et al., 2015; Oostwoud et al., 2016). Animals in the third group $(n=8)$ received intraperitoneal injections of saline $(1 \mathrm{ml} / \mathrm{kg})$ and served as a control.

A day after the sixth injection of a solution, to ensure that the drug was cleared from the brain, animals in each group were anesthetized (induction of $5 \%$ isoflurane; maintained at $2 \%$ isoflurane mixed with medical oxygen) and subjected to a PET/computed tomography (CT) scan with (5S,10R)-(+)-5-Methyl-10,11-dihydro-5H-dibenzo[a,d]cyclohepten5,10-imine (ABP-688), performed on two dedicated small-animal PET/CT scanners (docked PET/CT and Siemens Inveon multimodal PET/CT; Siemens Medical Solutions, Knoxville, TN) that had a reconstructed image resolution of approximately $1.2 \mathrm{~mm}$ at the center of the field of view. PET data were acquired for a total of 60 minutes in list-mode format (frames: $2 \times 10,3 \times 20,3 \times 30,3 \times 60,3 \times 150$, and $9 \times 300$ seconds) with ABP-688 injected immediately after the start of the PET acquisition. After the PET scan, a CT image was acquired in three different bed positions. Small-animal $\mu$ PET images were reconstructed by use of a two-dimensional ordered subset expectation maximization with four iterations and 16 subsets after Fourier rebinning. The $\mu$ PET images were then reconstructed on a $128 \times$ $128 \times 159$ grid with a voxel size of $0.776 \times 0.776 \times 0.776 \mathrm{~mm}$. Normalization, dead time correction, random subtraction, CT-based attenuation, and single-scatter simulation corrections were applied (Watson, 2000).

The day after the seventh injection, animals underwent an MRS scan. All magnetic resonance (MR)-based measurements were performed on a seven Tesla Pharmascan (Avance III) with a horizontal bore ( $16 \mathrm{~cm}$ in diameter; Bruker Biospin, Ettlingen, Germany) equipped with a standard Bruker coil setup using a quadrature volume resonator for excitation and array rat head surface coil for signal detection. The system was interfaced to a Linux personal computer running TopSpin 3.1 and ParaVision 6.0.1 software (Bruker Biospin).

All rats were initially anesthetized using 5\% isoflurane and maintained at $2 \%$ isoflurane in a mixture with medical oxygen. The volume of interest (VOI) for ${ }^{1} \mathrm{H}-\mathrm{MRS}$ was localized using multislice Rapid Acquisition with Refocused Echoes (RARE) images using a TurboRARE pulse sequence (Hennig et al., 1986). A VOI $(1.4 \times 2.2 \times$ $2.7 \mathrm{~mm}=8.32 \mathrm{~mm}^{3}$ ) was placed in the caudate putamen $(\mathrm{CP})$ of the rat brain, as shown in Fig. 2A, by using the Paxinos and Watson rat brain atlas (Paxinos, 2013) as a reference (between bregma \pm-0.24 and $\pm 1.92 \mathrm{~mm}$ ). More details regarding this procedure can be found in the Supplemental Materials.
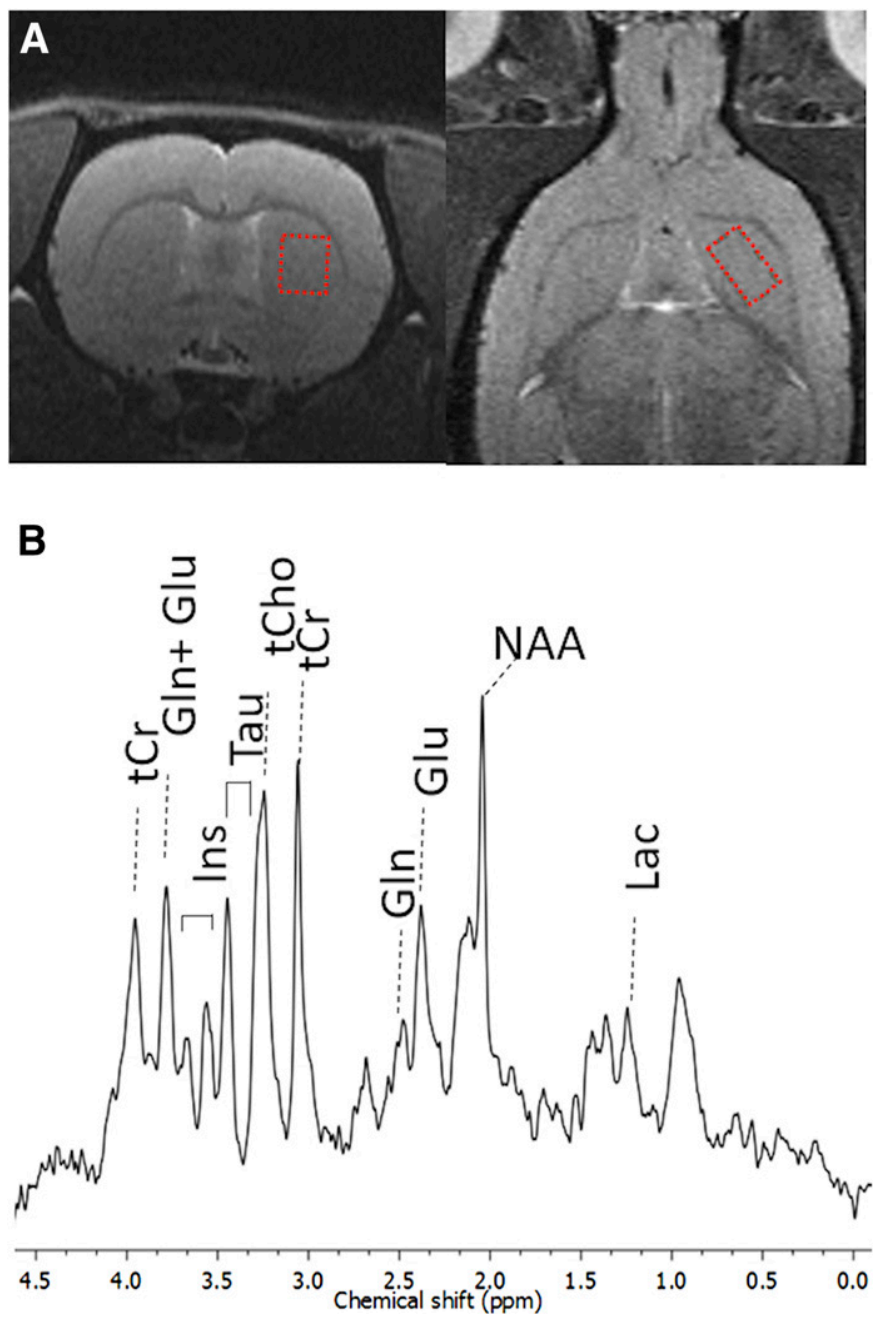

Fig. 2. (A) The VOI is positioned at the caudate putamen (indicated by the red boxes on $\mathrm{T} 2$ weighted axial and coronal images). (B) The major peaks are labeled on the representative spectra. Ins, inositol; Lac, lactate; NAA, $N$-acetylaspartate; PCho, Phospho-choline; Tau, taurine; total choline (tCho), Pcho + GPC; total creatine (tCr), $\mathrm{PCr}+\mathrm{Cr}$. The voxel size is $8.32 \mathrm{~mm}^{3}$. 
Following the MRS scan animals, were sacrificed and their brains were removed and stored at $-80^{\circ} \mathrm{C}$. To verify the results of the PET scan, the brains of the animals in each group were subjected to a histologic verification with an mGluR5 staining. In total, eight animals in the ebselen group were compared with nine animals in the MK-801 group for the immunohistochemistry analysis, as one brain removal did not succeed.

PET Analysis. Dynamic images were processed using PMOD 3.3 (PMOD Technologies, Zurich, Switzerland). After cropping, the static image corresponding to the time-averaged frames of each dynamic acquisition was spatially transformed to an in-house-developed rat brain template for ABP in a brain normalization step (Verhaeghe et al., 2014). The obtained transformation was then applied to the dynamic image frames to equally transform those to the same ABP template. Extracerebral activity was removed by applying a brain mask available from the software. Time-activity curves of the investigated regions were extracted from the resulting dynamic images and used as the input for the simplified reference tissue model (Lammertsma and Hume, 1996), with the cerebellum as the reference. This kinetic modeling step subsequently produced the average binding potential (BPnd) of the VOI of each region. Parametric BPnd maps were generated by pixelwise kinetic modeling using the simplified reference tissue model 2. These BPnd output values were investigated by means of a VOI-based analysis (with a predefined rat brain VOI template available in the software), using a Mann-Whitney test, to compare the two conditions (MK-801 vs. Ebselen) crosssectionally. Additionally, a voxel-based analysis was performed using statistical parametric mapping on the aforementioned parametric BPnd maps. These data were analyzed using an unpaired two-sample $t$ test with a cluster threshold of 125 voxels $\left(1 \mathrm{~mm}^{3}\right)$ and a significance level of 0.01 . The resulting $\mathrm{T}$ maps were again overlaid on the aforementioned brain VOI template delineating the different brain regions displaying significant increases (hyper) in a red-yellow format and significant decreases (hypo) in a blue-green format.

MR Analysis. In vivo ${ }^{1} \mathrm{H}$ MR spectra were analyzed using an automated deconvolution program (LCModel) (Provencher, 2001) as described previously (Orije et al., 2015). The LCModel analysis was performed within the chemical shift range of $0.2-4.2 \mathrm{ppm}$. The following metabolites were included in the basis set of the LCModel (Fig. 2B): alanine, aspartate, creatine (Cr), phosphocreatine (PCr), $\gamma$-aminobutyric acid, glucose, glutamate (Glu), glutamine (Gln), glutathione, glycerophosphorylcholine (GPC), phosphorylcholine, inositol, lactate, $N$-acetylaspartate, $N$-acetylaspartylglutamate, scyllo-inositol, and taurine. In addition to this standard set of brain metabolites, LCModel includes nine stimulated macromolecule and lipid peaks (Pfeuffer et al., 1999) as shown in Fig. 2B. The results of macromolecules and lipids were not included in the study. The reliability of the metabolite quantification was assessed using the Cramér-Rao lower bounds (CRLBs; estimated error of quantification) provided by LCModel (Table 1).

Poorly fitted metabolite peaks (CRLB of $>50 \%$ ) were not included in further analysis. Metabolites with CRLB $\leq 50 \%$ in at least $50 \%$ of the spectra were included in the neurochemical profile. Also, as neurometabolite levels fluctuate during the day, if the time of the MRS scan deviated from the originally foreseen time, the animal was not included in further analysis. This left us with a group size of 8 for the ebselen group, a group size of 10 for the MK-801 group, and a group size of 8 for the control group. A group correlation matrix for metabolites was created. If the correlation between two metabolites was consistently high (i.e., correlation coefficient higher than -0.5), the sum of the metabolites was reported (Provencher, 2001). We computed a correlation matrix for metabolites quantified for all animals to assess which metabolites could be reliably resolved from each other. If the negative correlation between two metabolites was very strong (i.e., correlation coefficient higher than -0.6), it was considered that these two metabolites cannot be individually quantified (Provencher, 2001). Therefore, their sum was reported. The amplitudes of $\mathrm{Cr}$ and $\mathrm{PCr}$ were strongly correlated: the corresponding matrix element equaled -0.9 . The amplitudes of phosphorylcholine and GPC were strongly correlated: the corresponding matrix element equaled -0.9 . For these metabolites, total creatine $(\mathrm{Cr}+\mathrm{PCr})$ and total choline (GPC) were reported. Due to the higher field, Glu and Gln could be quantified individually with good accuracy. Absolute concentrations (in micromoles per gram) of metabolites were determined relative to an unsuppressed water signal acquired from the same VOI (Minati et al., 2010). Further specifics on how these data were corrected can be found in the Supplemental Materials. For the purpose of this paper, we focused on the glutamate and glutamine levels and how these correlated with the other neurometabolites.

To estimate T2 maps of the $\mathrm{CP}$, a rectangular region of interest $\left( \pm 0.05 \mathrm{~cm}^{2}\right)$ was manually defined for the left and right CP separately using the Paxinos and Watson rat brain atlas (sixth edition) as a reference atlas. The data from left and right $\mathrm{CP}$ were averaged and recorded as the T2 relaxation time. The coronal images acquired with multi-slice multi-echo (MSME) sequence for T2 mapping were analyzed automatically with ParaVision 6.01 as described previously, using the image sequence analysis tool (Kara et al., 2015).

Immunohistochemistry. Coronal slices of the $\mathrm{CP}$ were made with a cryotome (Leica, Wetzlar, Germany) at a thickness of $20 \mu \mathrm{m}$ on Superfrost Plus slides (Thermo Scientific, Merelbeke, Belgium). These slices were stored at $-80^{\circ} \mathrm{C}$ until staining. The staining procedure can be found in the Supplemental Materials.

All pictures were taken with a Nikon Ti wide-field microscope (Nikon Instruments, Paris, France), digitalized by a Nikon Digital Sight DS-FI2 camera, using NIS Elements AR software, version 4.51.01 (Nikon Instruments).

TABLE 1

Metabolite concentrations (in micromoles per gram) and CRLB values

\begin{tabular}{lcccccc}
\hline \multirow{2}{*}{ Metabolite } & \multicolumn{3}{c}{ Concentration (mean \pm S.D.) } & & & \\
\cline { 2 - 4 } & Control $(n=8)$ & Ebselen $(n=8)$ & MK-801 $(n=10)$ & & & \\
\hline & $\mu \mathrm{mol} / \mathrm{g}$ & $\mu \mathrm{mol} / \mathrm{g}$ & $\mu \mathrm{mol} / \mathrm{g}$ & $\%$ & $\%$ & $\%$ \\
tCr & $6.1 \pm 0.5$ & $6.1 \pm 0.3$ & $6.0 \pm 0.5$ & 6 & 6 & 6 \\
GABA & $1.3 \pm 0.3$ & $1.5 \pm 0.2$ & $1.4 \pm 0.4$ & 29 & 24 & 26 \\
Glc & $1.3 \pm 0.5$ & $1.3 \pm 0.3$ & $1.4 \pm 0.2$ & 38 & 35 & 31 \\
Gln & $2.7 \pm 0.3$ & $2.2 \pm 0.4$ & $2.9 \pm 0.5$ & 18 & 22 & 18 \\
Glu & $8.4 \pm 0.7$ & $8.9 \pm 1.1$ & $8.8 \pm 0.3$ & 6 & 6 & 6 \\
tCh & $1.3 \pm 0.1$ & $1.4 \pm 0.2$ & $1.4 \pm 0.1$ & 9 & 8 & 8 \\
GSH & $1.1 \pm 0.2$ & $1.3 \pm 0.5$ & $1.4 \pm 0.4$ & 24 & 22 & 21 \\
Ins & $2.9 \pm 0.5$ & $2.7 \pm 0.7$ & $2.5 \pm 0.6$ & 14 & 16 & 17 \\
Lac & $1.3 \pm 0.5$ & $1.5 \pm 0.7$ & $1.4 \pm 0.3$ & 32 & 31 & 31 \\
NAA & $6.7 \pm 0.4$ & $6.4 \pm 0.4$ & $6.2 \pm 0.5$ & 6 & 6 & 6 \\
Tau & $9.0 \pm 0.5$ & $7.9 \pm 0.6$ & $8.3 \pm 0.7$ & 5 & 6 & 6 \\
\hline
\end{tabular}

Glc, glucose; GSH, glutathione; Ins, inositol; Lac, lactate; NAA, $N$-acetylaspartate; Tau, taurine; $\mathrm{tCh}$, total choline; $\mathrm{tCr}$, total creatine. 
Using ImageJ (version 1.47; National Institutes of Health, Bethesda, MD), mGluR5 receptor immunoreactivity was visualized. Analysis of the images consisted of the evaluation of the area fraction, which refers to the percentage of pixels in the image or selection that have been highlighted following a threshold, which was fixed at the same value for each image to eliminate any of the background.

\section{Results and Statistical Analyses}

Acute injection of MK-801 caused the animals of this group to display hyperactivity, consistent with what was previously described (Wu et al., 2005). No other unexpected erratic behavior was displayed over the course of the experiment. During the course of the scans, no aberrant behavior was observed. The average weight of the animals receiving this drug at the start of the experiment was $326.42 \pm 11.88 \mathrm{~g}$. At the end of the experiment, the average weight of these animals was significantly lower: $308.58 \pm 9.93 \mathrm{~g}$. Animals receiving ebselen had an average weight of $331.33 \pm 11.02 \mathrm{~g}$ at the beginning of the experiment, which significantly decreased to $322.17 \pm$ $11.21 \mathrm{~g}$ at the end of the experiment. Animals of the control group had a weight of $353.96 \pm 9.94 \mathrm{~g}$ at the scanning time point, which was significantly higher than the weight at the start of the experiment $(324.80 \pm 10 \mathrm{~g})$.

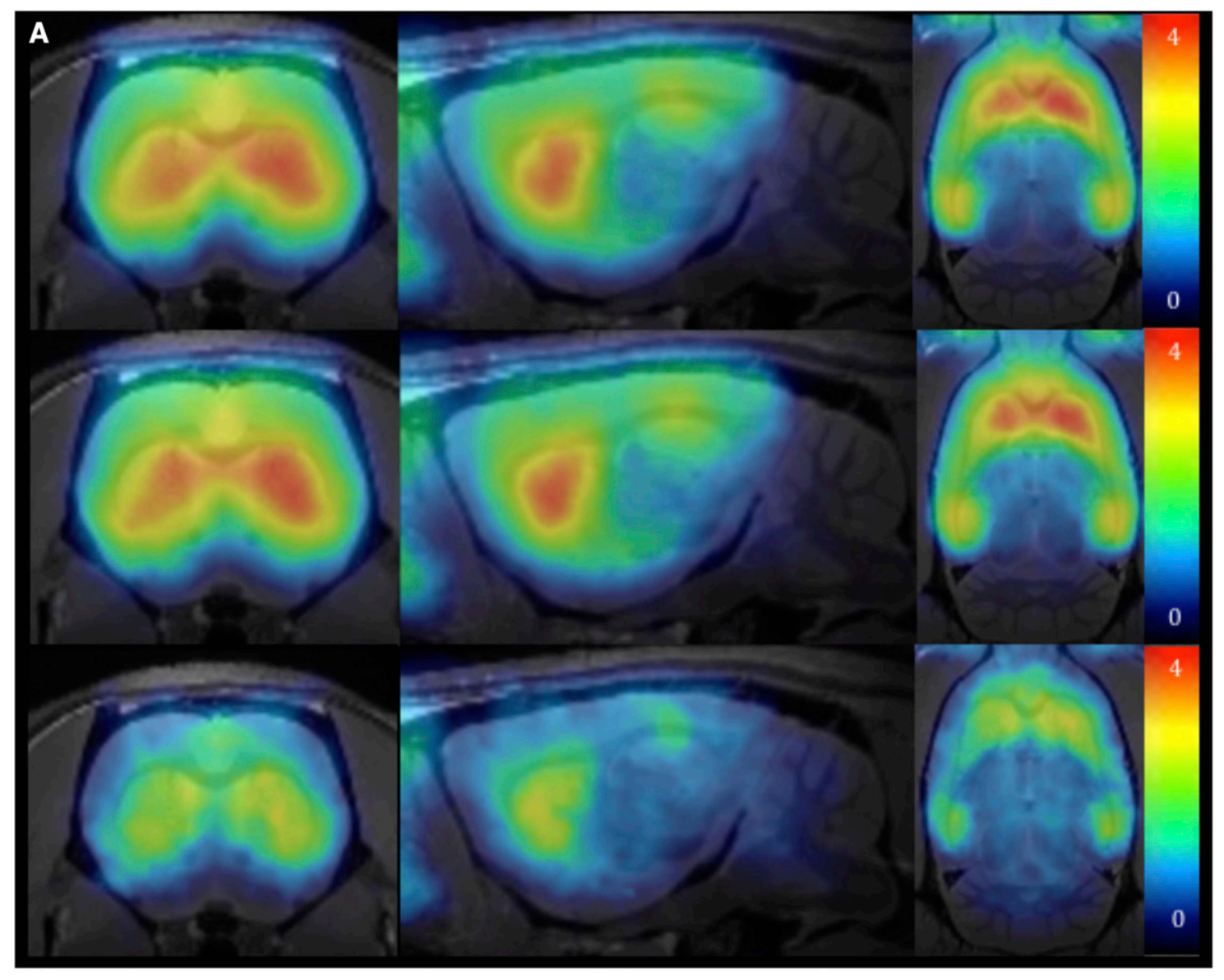

B

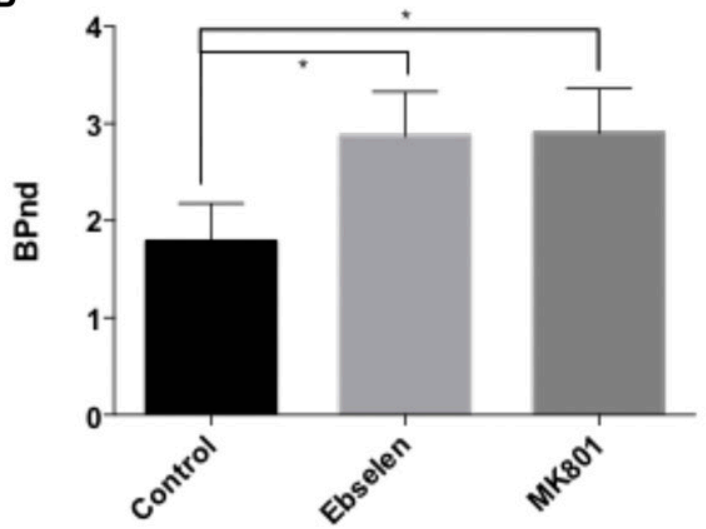

Fig. 3. (A) BPnd maps of ABP-688 for ebselen (top), MK-801 (middle), and saline (bottom) are displayed, with higher values displayed in red and lower values in a green-blue format. (B) BPnd values plotted showing the difference between the different groups. 
PET Data. VOI-based analysis revealed a significant difference when comparing the BPnd in the CP between the control animals $(1.97 \pm 0.39)$ and the animals receiving MK-801 (2.90 \pm 0.47 , constituting an increase of $47.21 \% \pm$ $17.00 \%)$ or ebselen $(2.87 \pm 0.46$, constituting an increase of $45.69 \% \pm 16.37 \%$ ) (Fig. 3).

This was confirmed with a voxel-based analysis $(P=0.01$, cluster size $=125$ voxels) investigating all regions using statistical parametric mapping, displaying strong significant $(P<0.01)$ increases after either drug in the frontal regions of the brain, in particular the majority of the $\mathrm{CP}$, the cortex, hippocampus, and amygdala. Furthermore, there were significant $(P<0.01)$ decreases in the majority of the cerebellum (Fig. 4).

Immunohistochemistry. Analysis revealed a significant $(P<0.05)$ difference in area percentage in the CP between control and ebselen, with an area fraction of $60.83 \% \pm 6.30 \%$ for ebselen and $50.21 \% \pm 5.71 \%$ for saline (Fig. 5). MK- $801 \mathrm{had}$ an area fraction of $57.14 \% \pm 9.23 \%$, which was not significantly different but with a tendency to increase.

MRS Data. No significant changes in glutamate level were found when comparing chronic ebselen and chronic MK-801 to control. However, a significant difference $(P<0.05)$ in the levels of glutamine between the ebselen and control animals was found. Animals treated with ebselen had a mean glutamine concentration of $2.2 \pm 0.4 \mu \mathrm{mol} / \mathrm{g}$, which was $18.52 \% \pm$ $5.42 \%$ lower than animals in the control group who had a mean of $2.7 \pm 0.3 \mu \mathrm{mol} / \mathrm{g}$. Animals of the MK-801 group had a mean of $2.9 \pm 0.5 \mu \mathrm{mol} / \mathrm{g}$, which was $7.41 \% \pm 2.10 \%$ higher than the control animals. Also, when considering the ratio of glutamate versus glutamine, a significant difference $(P<0.01)$ was present between the ebselen and control groups with a mean of $4.1 \pm 0.6$ for ebselen, which was $32.26 \% \pm 8.88 \%$ higher than the ratio for the control animals $(3.1 \pm 0.4$; Fig. 6$)$. This ratio was correlated $(P<0.05)$ with glutamine but not with glutamate.

A correlation between the MRS signal and the BPnd of the CP revealed no significant correlation with any of the neurometabolites $(P>0.05)$.

The mean $\mathrm{T} 2$ values of the $\mathrm{CP}$ of the rats receiving ebselen and MK-801 were not statistically significant compared to the rats receiving control $(P<0.05$, two-tailed Student's test, $n=8$ for each group). The values were estimated as $40.76 \pm$ $0.74 \mathrm{~ms}, 40.95 \pm 0.80 \mathrm{~ms}$, and $41.255 \pm 0.68 \mathrm{~ms}$ for the groups exposed to MK-801, ebselen, and control, respectively.

\section{Discussion}

This study evaluated the potential of both ebselen and MK-801 to affect the glutamate receptors and neurotransmitter levels in healthy naïve rats, as possible modulators for the preclinical QP-induced OCD model. Previous work established that ebselen has a direct effect on the glutamatergic system by reducing the $\mathrm{K}^{+}$-evoked release of glutamate (Nogueira et al., 2002; Moretto et al., 2007), while low dosages of MK-801 selectively block NMDA receptors in the GABAergic interneurons, thus increasing glutamate release (Xi et al., 2009). A previous study performed by our laboratory confirmed glutamate increased when MK-801 was given acutely. Other NMDA antagonists, such as ketamine, when administered acutely, also increase glutamate (Wyckhuys et al., 2013).

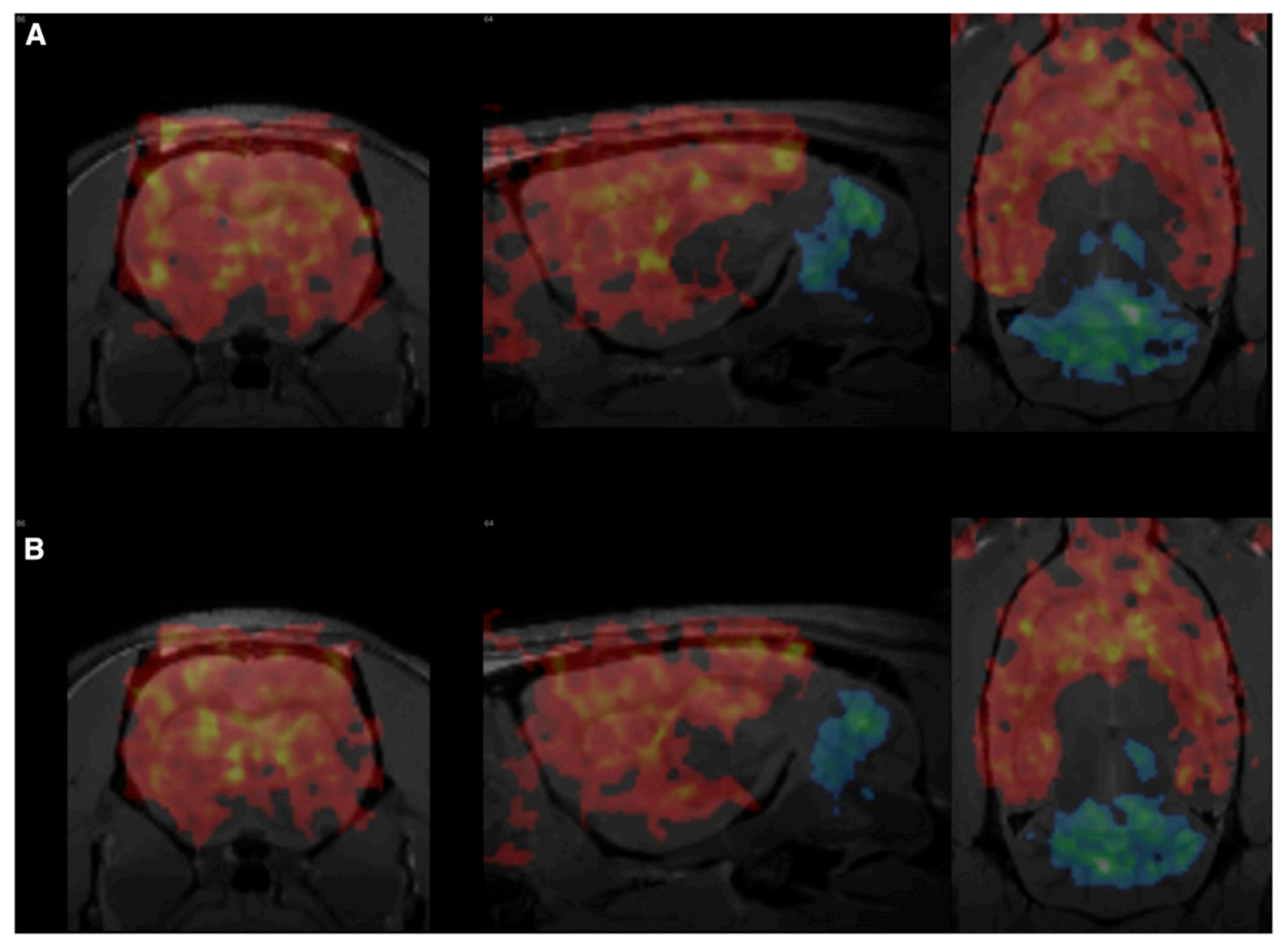

Fig. 4. Statistical parametric mapping maps of ABP-688 for both ebselen (A) and MK-801 (B) versus saline, with significantly higher $t$ values (hypermetabolism) displayed in red and significantly lower $t$ values (hypometabolism) in a blue-green format. 


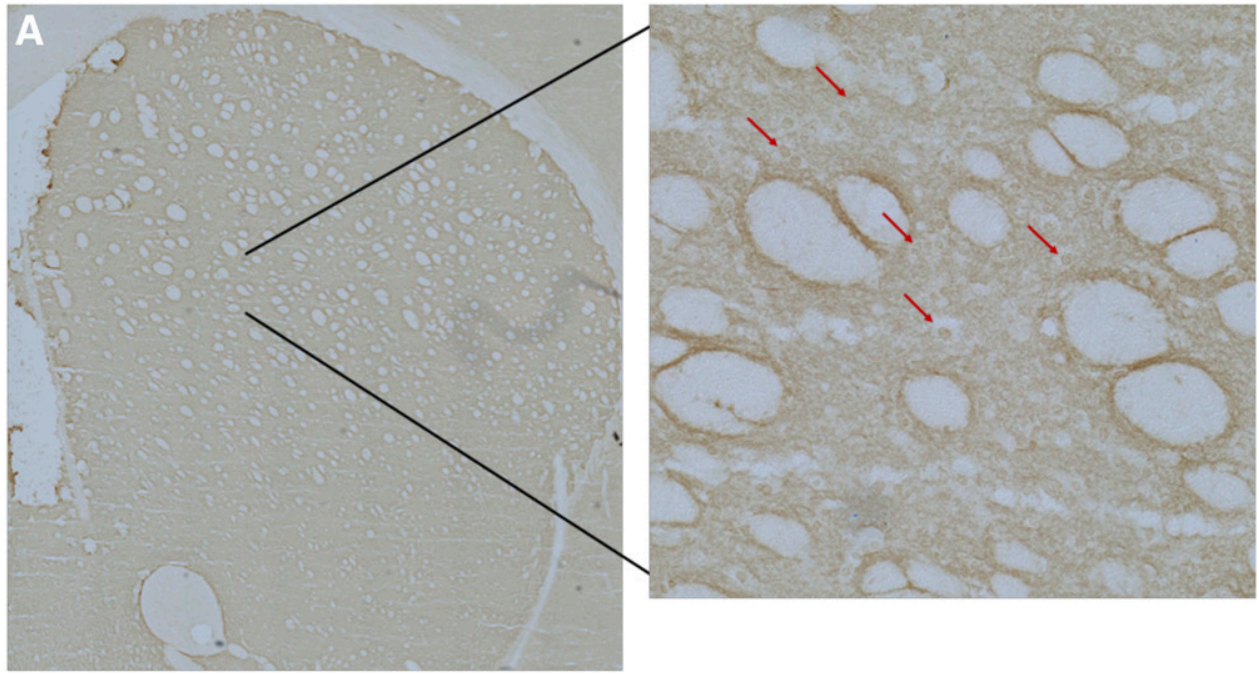

B

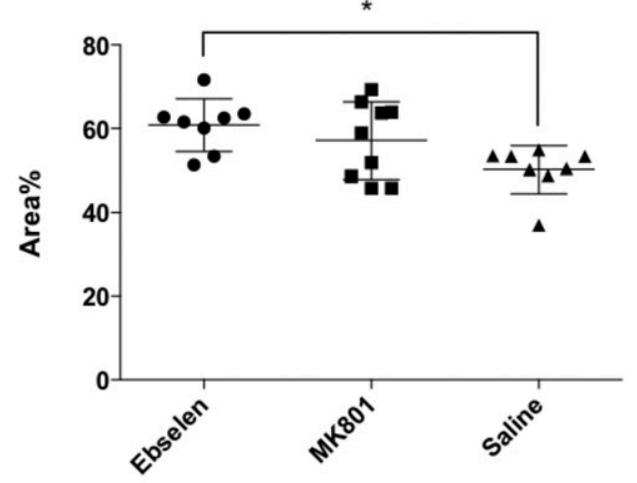

Fig. 5. (A) Overview image of the CP after immunohistochemistry (original magnification, $20 \times$ ) with the mGluR5 antibody. Arrows point to cells with densely stained membranes rich in mGluR5. (B) Investigated parameter of the immunohistochemistry is presented here (area percentage) after chronic ebselen $(n=8)$, chronic MK-801 $(n=9)$, and saline $(n=8)$. Individual points, median, and S.D. of each group are displayed. (C) Overview images of the three different conditions (left to right: ebselen, MK-801, saline). $* P<0.05$.

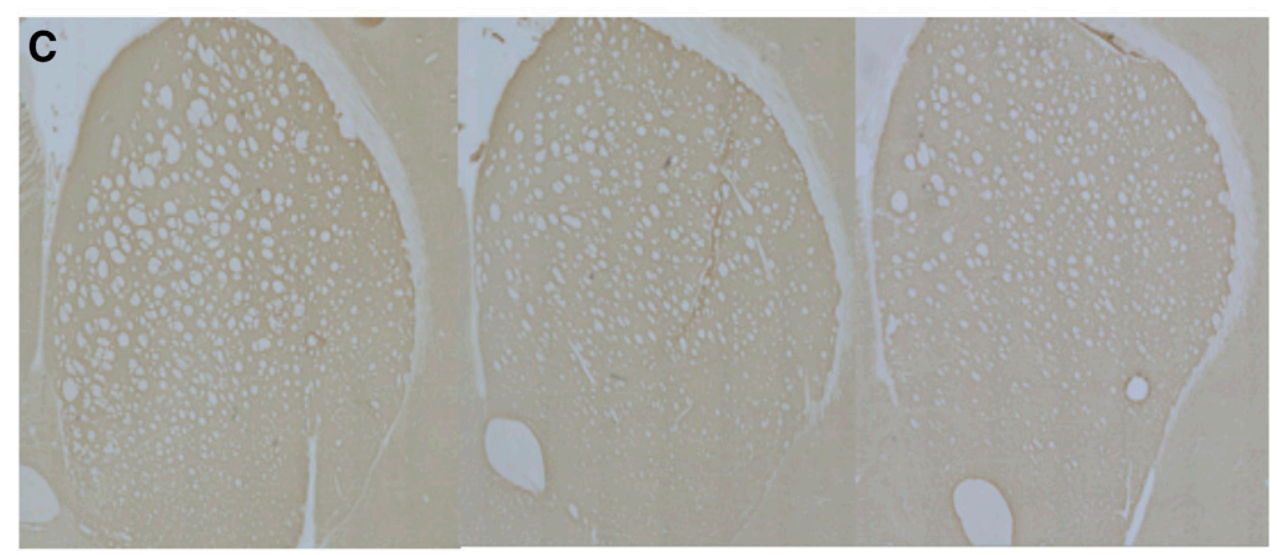

In contrast, MK-801 appears to decrease glutamate levels when administered chronically, which is potentially caused by chronic blockade of the NMDA receptor, making non-NMDA Glu receptors discompensatory and decreasing the terminal excitability of glutamatergic neurons, resulting in an attenuation of the Glu levels (Tsukada et al., 2005; Zuo et al., 2006). Alternatively, this decrease of Glu might also be caused by postsynaptic inhibition of other neuronal systems to NMDA receptor-mediated neurotransmission (Tsukada et al., 2005; Zuo et al., 2006). Another explanation can be found in the upregulation of Glu transporters (Tsukada et al., 2005; Zuo et al., 2006).
After chronic ebselen, the increase in mGluR5 area fraction measured by IHC was significant, indicating more mGluR5 distribution, possibly as a result of chronically less endogenous glutamate. For MK-801, a similar trend is noticeable, albeit not significant with the current power.

Also, the PET/CT results showed significant increases in BPnd after chronic administration of both ebselen and MK-801, also indicating more availability of the mGluR5 receptor. MK-801 is not the only NMDA antagonist that has been investigated by ABP-688. Previously, a clinical study used this tracer to investigate the acute effect of ketamine $(0.23 \mathrm{mg} / \mathrm{kg}$ over 1 minute, then $0.58 \mathrm{mg} / \mathrm{kg}$ over 1 hour) with 

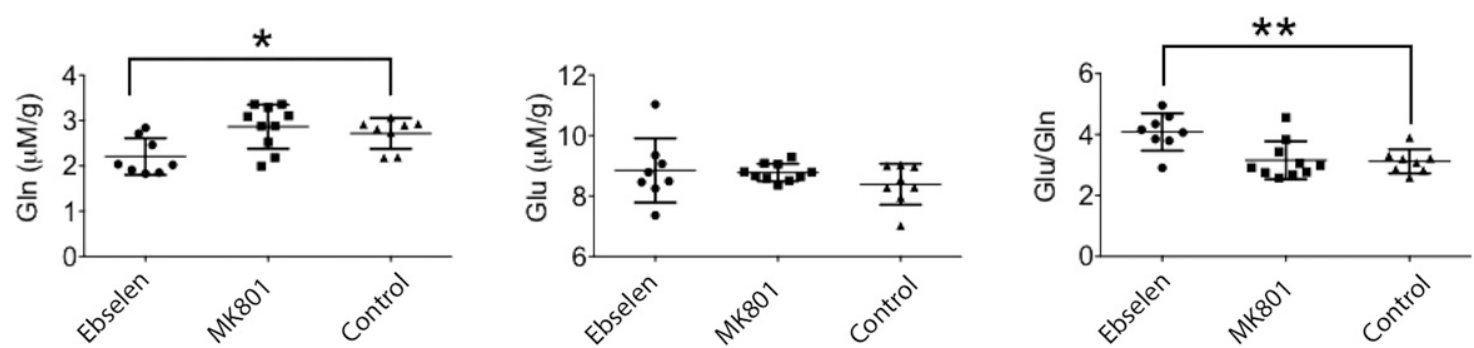

Fig. 6. The investigated MRS parameters (glutamate, glutamine, and the ratio between the two) extracted from the MRS spectrum are plotted here for both ebselen $(n=8)$ and MK-801 $(n=10)$. Individual points, median, and S.D. of the group are displayed. ${ }^{*} P<0.05 ; * * P<0.01$.

the intention of increasing Glu, and reported significant decreases in BPnd. As ABP-688 binds at different sites on the receptor and hence does not compete with glutamate, at first sight one could hypothesize that glutamate fluctuations do not influence the BPnd. However, it should be noted that the affinity of the receptor for the tracer is in part determined by the endogenous glutamate. For example, DeLorenzo et al. (2015) used $N$-acetylcysteine, which is known to increase extracellular glutamate, and reported decreases in BPnd. Similarly, Zimmer et al. (2015) used ceftriaxone to decrease extracellular levels of glutamate and reported increases in the BPnd of ABP-688. Therefore, our results, displaying strong increases in BPnd after treatment with ebselen or MK-801 when compared with control, indicate lower levels of extracellular glutamate that positively affect the affinity of the tracer for mGluR5, consistent with previous data (Tsukada et al., 2005; Zuo et al., 2006). Thus, it is possible that the increase in mGluR5 distribution in the PET/CT results was overestimated due to the increase in tracer affinity caused by a decrease in extracellular glutamate. Alternatively, as NMDA and mGluR5 are interconnected in the brain, it is plausible for the changes induced by MK- 801 to be present only on the level of receptor interaction and not on glutamate release.

In contrast to the results from the PET/CT and immunohistochemistry, indicating decreased levels of glutamate, the MRS scan showed that the chronic exposure to either drug did not affect glutamate levels in such a way that it differed from the control condition in the visualized time frame. When considering the PET/CT and immunohistochemistry results, this seems to contradict the hypothesis of a decrease in glutamate. However, it is important to note that MRS visualizes the total glutamate pool as it does not discriminate between intracellular and extracellular neurochemicals. The concentration of the neurochemicals is mostly driven by the intracellular concentration due to the negligible volume of the extracellular space. Thus, it could be that the release of glutamate in the extracellular space is, in fact, changed, directly affecting the glutamate receptors as visualized by $\mathrm{PET} / \mathrm{CT}$ and immunohistochemistry. Interestingly, when considering this flux of Glu and Gln, there were significant decreases in Gln after chronic injection with ebselen. Because animals are repeatedly exposed to the drug, certain compensatory changes could occur. As glutamate is readily converted into glutamine through the glutamine synthetase pathway by astrocytes, it is possible that, due to decreased glutamate levels after chronic administration of ebselen, the available glutamine was converted into glutamate to preserve a normal level of glutamate, thereby depleting the Gln pool to compensate for this as the acute effect of ebselen wore off. Because the amount of glutamate present in the synapse is maintained homeostatically at a low level at all times, with $85 \%$ of glutamate being converted to glutamine (Hertz, 2013) and ebselen acutely decreasing Glu levels, this could explain the distorted balance in the Glu/Gln ratio after administration of ebselen. Although this ratio had a tendency to increase after chronic administration of MK-801, this was not significant in this particular group.

In conclusion, both ebselen and MK-801 affect the mGluR5 receptor when administered chronically in the dosages described in this study. Although no direct effects on total glutamate were visualized with MRS, the changes in glutamine do hint toward changes in the total Glu-Gln pool.

Taken together, these two drugs are interesting modulators for further investigation in glutamatergic pathologies, such as obsessive compulsive disorder, Fragile X, epilepsy, and autism, and for further elucidation of their working mechanisms to optimize their use and potential.

\section{Acknowledgments}

We thank Philippe Joye, Caroline Berghmans, and Annemie Van Eetveldt for their expertise and technical assistance.

\section{Authorship Contributions}

Participated in research design: S. Servaes, Kara, Glorie, S. Staelens. Conducted experiments: S. Servaes, Kara.

Performed data analysis: S. Servaes, Kara.

Wrote or contributed to the writing of the manuscript: S. Servaes, Kara, Van Der Linden, S. Stroobants, S. Staelens.

\section{References}

Ade KK, Wan Y, Hamann HC, O'Hare JK, Guo W, Quian A, Kumar S, Bhagat S, Rodriguiz RM, Wetsel WC, et al. (2016) Increased metabotropic glutamate receptor 5 signaling underlies obsessive-compulsive disorder-like behavioral and striatal circuit abnormalities in mice. Biol Psychiatry 80:522-533.

Albelda N, Bar-On N, and Joel D (2010) The role of NMDA receptors in the signal attenuation rat model of obsessive-compulsive disorder. Psychopharmacology (Berl) 210:13-24.

Arnold PD, Rosenberg DR, Mundo E, Tharmalingam S, Kennedy JL, and Richter MA (2004) Association of a glutamate (NMDA) subunit receptor gene (GRIN2B) with obsessive-compulsive disorder: a preliminary study. Psychopharmacology (Berl) 174:530-538.

Azad GK and Tomar RS (2014) Ebselen, a promising antioxidant drug: mechanisms of action and targets of biological pathways. Mol Biol Rep 41:4865-4879.

Bloch MH, Wasylink S, Landeros-Weisenberger A, Panza KE, Billingslea E, Leckman JF, Krystal JH, Bhagwagar Z, Sanacora G, and Pittenger C (2012) Effects of ketamine in treatment-refractory obsessive-compulsive disorder. Biol Psychiatry 72: 964-970.

Chakrabarty K, Bhattacharyya S, Christopher R, and Khanna S (2005) Glutamatergic dysfunction in OCD. Neuropsychopharmacology 30:1735-1740.

Chana G, Laskaris L, Pantelis C, Gillett P, Testa R, Zantomio D, Burrows EL, Hannan AJ, Everall IP, and Skafidas E (2015) Decreased expression of mGluR5 within the dorsolateral prefrontal cortex in autism and increased microglial number in mGluR5 knockout mice: pathophysiological and neurobehavioral implications. Brain Behav Immun 49:197-205.

Coyle JT (2006) Glutamate and schizophrenia: beyond the dopamine hypothesis. Cell Mol Neurobiol 26:365-384. 
Dalla Corte CL, Bastos LL, Dobrachinski F, Rocha JBT, and Soares FAA (2012) The combination of organoselenium compounds and guanosine prevents glutamateinduced oxidative stress in different regions of rat brains. Brain Res 1430:101-111.

DeLorenzo C, DellaGioia N, Bloch M, Sanacora G, Nabulsi N, Abdallah C, Yang J, Wen R, Mann JJ, Krystal JH, et al. (2015) In vivo ketamine-induced changes in $\left[{ }^{11} \mathrm{C}\right] \mathrm{ABP} 688$ binding to metabotropic glutamate receptor subtype 5. Biol Psychiatry 77:266-275.

Denys D, Mantione M, Figee M, van den Munckhof P, Koerselman F, Westenberg H, Bosch A, and Schuurman R (2010) Deep brain stimulation of the nucleus accumbens for treatment-refractory obsessive-compulsive disorder. Arch Gen Psychiatry 67:1061-1068.

Ghasemi M and Schachter SC (2011) The NMDA receptor complex as a therapeutic target in epilepsy: a review. Epilepsy Behav 22:617-640.

Hennig J, Nauerth A, and Friedburg H (1986) RARE imaging: a fast imaging method for clinical MR. Magn Reson Med 3:823-833.

Hertz L (2013) The glutamate-glutamine (GABA) cycle: importance of late postnatal development and potential reciprocal interactions between biosynthesis and degradation. Front Endocrinol (Lausanne) 4:59.

Hillhouse TM and Porter JH (2014) Ketamine, but not MK-801, produces antidepressant-like effects in rats responding on a differential-reinforcement-oflow-rate operant schedule. Behav Pharmacol 25:80-91.

Kara F, Höfling C, Roßner S, Schliebs R, Van der Linden A, Groot HJM, and Alia A (2015) In vivo longitudinal monitoring of changes in the corpus callosum integrity during disease progression in a mouse model of Alzheimer's disease. Curr Alzheimer Res 12:941-950.

Kelly E, Schaeffer SM, Dhamne SC, Lipton JO, Lindemann L, Honer M, Jaeschke G, Super CE, Lammers SH, Modi ME, et al. (2018) mGluR5 modulation of behaviora and epileptic phenotypes in a mouse model of tuberous sclerosis complex. Neuropsychopharmacology 43:1457-1465.

Kosten L, Verhaeghe J, Verkerk R, Thomae D, De Picker L, Wyffels L, Van Eetveldt A, Dedeurwaerdere S, Stroobants S, and Staelens S (2016) Multiprobe molecular imaging of an NMDA receptor hypofunction rat model for glutamatergic dysfunction. Psychiatry Res Neuroimaging 248:1-11.

Lammertsma AA and Hume SP (1996) Simplified reference tissue model for PET receptor studies. Neuroimage 4:153-158.

Lee EJ, Choi SY, and Kim E (2015) NMDA receptor dysfunction in autism spectrum disorders. Curr Opin Pharmacol 20:8-13.

McGrath MJ, Campbell KM, Parks CR, and Burton FH (2000) Glutamatergic drugs exacerbate symptomatic behavior in a transgenic model of comorbid Tourette's syndrome and obsessive-compulsive disorder. Brain Res 877:23-30.

Meldrum BS (2000) Glutamate as a neurotransmitter in the brain: review of physiology and pathology. J Nutr 130 (4S Suppl):1007S-1015S.

Miladinovic T, Nashed MG, and Singh G (2015) Overview of glutamatergic dysregulation in central pathologies. Biomolecules 5:3112-3141

Minati L, Aquino D, Bruzzone MG, and Erbetta A (2010) Quantitation of normal metabolite concentrations in six brain regions by in-vivoH-MR spectroscopy. J Med Phys 35:154-163.

Moretto MB, Thomazi AP, Godinho G, Roessler TM, Nogueira CW, Souza DO, Wofchuk S, and Rocha JBT (2007) Ebselen and diorganylchalcogenides decrease in vitro glutamate uptake by rat brain slices: prevention by DTT and GSH. Toxicol In Vitro 21:639-645.

Nogueira CW, Rotta LN, Zeni G, Souza DO, and Rocha JBT (2002) Exposure to ebselen changes glutamate uptake and release by rat brain synaptosomes. $\mathrm{Neu}$ rochem Res 27:283-288.

Oostwoud LC, Gunasinghe P, Seow HJ, Ye JM, Selemidis S, Bozinovski S, and Vlahos R (2016) Apocynin and ebselen reduce influenza A virus-induced lung inflammation in cigarette smoke-exposed mice. Sci Rep 6:20983.

Orije J, Kara F, Guglielmetti C, Praet J, Van der Linden A, Ponsaerts P, and Verhoye $M$ (2015) Longitudinal monitoring of metabolic alterations in cuprizone mouse model of multiple sclerosis using $1 \mathrm{H}$-magnetic resonance spectroscopy. Neuroimage 114:128-135

Ozyigit F, Kucuk A, Akcer S, Tosun M, Kocak FE, Kocak C, Kocak A, Metineren H, and Genc O (2015) Different dose-dependent effects of ebselen in sciatic nerve ischemia-reperfusion injury in rats. Bosn J Basic Med Sci 15:36-43.

Paxinos G and Watson C (2013) The Rat Brain in Stereotaxic Coordinates. Academic Press (7th Edition), Cambridge, Massachusetts, United States.

Pfeuffer J, Tkác I, Provencher SW, and Gruetter R (1999) Toward an in vivo neurochemical profile: quantification of 18 metabolites in short-echo-time (1)H NMR spectra of the rat brain. J Magn Reson 141:104-120.

Pittenger C, Bloch MH, and Williams K (2011) Glutamate abnormalities in obsessive compulsive disorder: neurobiology, pathophysiology, and treatment. Pharmaco Ther 132:314-332.
Pop AS, Gomez-Mancilla B, Neri G, Willemsen R, and Gasparini F (2014) Fragile $\mathrm{X}$ syndrome: a preclinical review on metabotropic glutamate receptor 5 (mGluR5) antagonists and drug development. Psychopharmacology (Berl) 231:1217-1226

Porciúncula LO, Rocha JBT, Boeck CR, Vendite D, and Souza DO (2001) Ebselen prevents excitotoxicity provoked by glutamate in rat cerebellar granule neurons. Neurosci Lett 299:217-220.

Provencher SW (2001) Automatic quantitation of localized in vivo $1 \mathrm{H}$ spectra with LCModel. NMR Biomed 14:260-264.

Rodriguez CI, Kegeles LS, Levinson A, Feng T, Marcus SM, Vermes D, Flood P, and Simpson HB (2013) Randomized controlled crossover trial of ketamine in obsessive-compulsive disorder: proof-of-concept. Neuropsychopharmacology 38: 2475-2483.

Schewe T (1995) Molecular actions of Ebselen-an antiinflammatory antioxidant. Gen Pharmacol Vasc Syst 26:1153-1169.

Seo JY, Lee CH, Cho JH, Choi JH, Yoo KY, Kim DW, Park OK, Li H, Choi SY, Hwang IK, et al. (2009) Neuroprotection of ebselen against ischemia/reperfusion injury involves GABA shunt enzymes. J Neurol Sci 285:88-94.

Servaes S, Glorie D, Verhaeghe J, Stroobants S, and Staelens S (2017) Preclinical molecular imaging of glutamatergic and dopaminergic neuroreceptor kinetics in obsessive compulsive disorder. Prog Neuropsychopharmacol Biol Psychiatry 77: $90-98$.

Shannon HE and Love PL (2004) Within-session repeated acquisition behavior in rats as a potential model of executive function. Eur $J$ Pharmacol 498:125-134.

Sleigh J, Harvey M, Voss L, and Denny B (2014) Ketamine - more mechanisms of action than just NMDA blockade. Trends Anaesth Crit Care 2014:76-81.

Thomas AG, Rojas C, Tanega C, Shen M, Simeonov A, Boxer MB, Auld DS, Ferraris DV, Tsukamoto T, and Slusher BS (2013) Kinetic characterization of ebselen, chelerythrine and apomorphine as glutaminase inhibitors. Biochem Biophys Res Commun 438:243-248.

Tsukada H, Nishiyama S, Fukumoto D, Sato K, Kakiuchi T, and Domino EF (2005) Chronic NMDA antagonism impairs working memory, decreases extracellular dopamine, and increases D1 receptor binding in prefrontal cortex of conscious monkeys. Neuropsychopharmacology 30:1861-1869.

Verhaeghe J, Wyffels L, Wyckhuys T, Stroobants S, and Staelens S (2014) Rat brain normalization templates for robust regional analysis of [11C]ABP688 positron emission tomography/computed tomography. Mol Imaging 13.

Watson CC (2000) New, faster, image-based scatter correction for 3D PET. IEEE Trans Nucl Sci 47:1587-1594.

Welch JM, Lu J, Rodriguiz RM, Trotta NC, Peca J, Ding J-D, Feliciano C, Chen M, Adams JP, Luo J, et al. (2007) Cortico-striatal synaptic defects and OCD-like behaviours in Sapap3-mutant mice. Nature 448:894-900.

Wu J, Zou H, Strong JA, Yu J, Zhou X, Xie Q, Zhao G, Jin M, and Yu L (2005) Bimodal effects of MK-801 on locomotion and stereotypy in C57BL/6 mice. Psychopharmacology (Berl) 177:256-263.

Wyckhuys T, Verhaeghe J, Wyffels L, Langlois X, Schmidt M, Stroobants S, and Staelens S (2013) N-acetylcysteine- and MK-801-induced changes in glutamate levels do not affect in vivo binding of metabotropic glutamate 5 receptor radioligand 11C-ABP688 in rat brain. J Nucl Med 54:1954-1961.

Xi D, Zhang W, Wang HX, Stradtman GG, and Gao WJ (2009) Dizocilpine (MK-801) induces distinct changes of $\mathrm{N}$-methyl-D-aspartic acid receptor subunits in parvalbumin-containing interneurons in young adult rat prefrontal cortex. Int $J$ Neuropsychopharmacol 12:1395-1408.

Yatmaz S, Seow HJ, Gualano RC, Wong ZX, Stambas J, Selemidis S, Crack PJ, Bozinovski S, Anderson GP, and Vlahos R (2013) Glutathione peroxidase-1 reduces influenza A virus-induced lung inflammation. Am J Respir Cell Mol Biol 48:17-26.

Zimmer ER, Parent MJ, Leuzy A, Aliaga A, Aliaga A, Moquin L, Schirrmacher ES, Soucy J-P, Skelin I, Gratton A, et al. (2015) Imaging in vivo glutamate fluctuations with [(11)C]ABP688: a GLT-1 challenge with ceftriaxone. J Cereb Blood Flow Metab 35:1169-1174.

Zuo DY, Zhang YH, Cao Y, Wu CF, Tanaka M, and Wu YL (2006) Effect of acute and chronic MK-801 administration on extracellular glutamate and ascorbic acid release in the prefrontal cortex of freely moving mice on line with open-field behavior. Life Sci 78:2172-2178.

Address correspondence to: Steven Staelens, Molecular Imaging Center Antwerp, University of Antwerp, Universiteitsplein 1, Wilrijk, Antwerp 2610, Belgium. E-mail: steven.staelens@uantwerpen.be 